\title{
Development of Optical pH Sensor using Conducting Polyaniline -Wrapped Multiwalled Carbon Nanotubes (PANI-MWCNTs) Nanocomposite
}

\author{
Milind V. Kulkarni* and Bharat B. Kale \\ Nanocomposite Laboratory, \\ Centre for Materials for Electronics Technology (C-MET), \\ Department of Information Technology, Govt. of India \\ Panchawati, Off Pashan Road, \\ Pune 411 008, INDIA \\ milindcmet@yahoo.com \\ milind@cmet.gov.in
}

\begin{abstract}
Highly transparent and water soluble nanocomposite of conducting Polyaniline wrapped - Multi-Walled Carbon Nanotubes (PANI - MWCNTs) was synthesized by an in-situ chemical polymerization method using sulphonic acid as a dopant. MWCNTs were functionalized prior to their use and then polymerized using ammonium per sulphate as an oxidizing agent. The nanocomposite was subjected for physico-chemical characterization using spectroscopic (UV-Vis and FT-IR), FE-SEM and thermal analysis. The UV-Visible spectrum of the salt phase (dark green) of the PANI-MWCNTs nanocomposite shows a free carrier tail with increasing absorption at higher wavelength, which confirms the presence of conducting emeraldine salt phase of the polyaniline and is further supported by FT-IR analysis. However, the base form (deep blue) of the nanocomposite shows a sharp peak at $600 \mathrm{~nm}$ representing an insulating pernigraniline phase of the polymer. The FE-SEM image shows the uniform wrapping/coating of the polyaniline over functionalized MWCNTs. The synthesized nanocomposite was then successfully used for the determination of $\mathrm{pH}$ of the solution $(\mathrm{pH} \mathrm{1-12)}$ and found to be a potential candidate for optical $\mathrm{pH}$ sensing.
\end{abstract}

Keywords: Nanocomposite, Polyaniline-MWCNTs, Spectroscopy, pH sensor.

\section{Introduction}

CNT has attracted a great deal of interest, both as advanced reinforcement $[1,2]$ and in a wide range of electronic industrial applications [3]. Its graphite structure with high-chemical stability during synthesis, its mechanical endurance under ultrasonication, and its electrical conductivity make CNT well suited to synthesizing a PANI/CNT composite. However, pristine CNT has no functional group to develop dispersibility in organic polar solvents and a polar interaction between the CNT and the polymer matrix. Thus, specific modifications were required through vigorous oxidation methods on the surface of the CNT, such as acid treatment or plasma treatment.

Polyaniline (PANI), an interesting conducting polymer, has been known for more than four decades, and is still of interest both academically and industrially. Because of its unique processibility together with the availability of relatively inexpensive monomer and high yield of polymerization, it has become one of the most technologically important polymeric materials. To improve the electrical, magnetic, and optical properties of CNTs, conjugated or conducting polymers are attached to their surfaces by in-situ polymerization. Among the various conducting polymers, PANI is of particular interest in synthesizing polymer-MWCNTs composites because of its environmental stability, good processability, and reversible control of the conductivity $[4,5]$.

Recently, functionalized multi walled carbon nanotubes ( $f$-MWCNTs)-Polyaniline (PANI) nanocomposite has attracted much attention due to their enhanced electronic properties [6] They are promising nanocomposite for new applications in chemistry, physics and particularly in development of new devices such as hydrogen storage, supercapacitor, biosensors, electromechanical actuators, 
nanoprobes for high-resolution imaging and many more $[7,8]$.

A typical method to prepare PANI/Multiwalled carbon nanotube (MWCNT) composites is an in - situ aqueous solution polymerization or emulsion polymerization $[9,10]$.

There is an advantage of an in-situ polymerization of $f$-MWCNTs to obtain good homogeneity which is lacking in an ex-situ polymerization. Hence, the attempts have been made to f-MWCNTs by in-situ polymerization.

The objective of this work is to demonstrate the covalently functionalization of multiwalled carbon nanotube (MWCNTs) and its utilization for the synthesis of PANI-MWCNTs nanocomposite via an in-situ polymerization with attractive physico-electrochemical properties. The nanocomposite was then characterized by using UV-Vis, and FT-IR spectroscopy. Morphology of the nanocomposite was studied by using FE-SEM analysis and the synthesized nanocomposite was then successfully used for optical $\mathrm{pH}$ sensing.

\section{Experimental}

\section{i) Materials}

Commercial MWCNTs (diameter 20-35 nm, length $10-50 \mu \mathrm{m}$ ) with a purity of $>95 \%$ was used as received. Aniline monomer (99\% purity,) was distilled under reduced pressure and stored at low temperature prior to use. Oxidizing agent, ammonium per sulfate (APS) and protonating acid, Dodecylbenzene sulphonic acid (DBSA) is of analytical reagent (AR) grade and used as received. All the solutions were prepared using double distilled water.

\section{ii) Functionalisation of MWCNTs}

The MWCNTs was first ultrasonicated using mixture of concentrated sulfuric acid $\left(\mathrm{H}_{2} \mathrm{SO}_{4}\right)$ and nitric acid $\left(\mathrm{HNO}_{3}\right)$ in a ratio of $3: 1$ for 8 hours at room temperature. After sonication in acid, MWCNTs were centrifuged and washed repeatedly with distilled water till the $\mathrm{pH}$ was neutral. The product was dried in an oven at $150^{\circ} \mathrm{C}$ for $2-3$ hours. Fine black powder was obtained and denoted as $f$-MWCNTs (functionalized-MWCNTs).

\section{iii) Synthesis of acid doped PANI-MWCNTs nanocomposite}

The conducting nanocomposite of PANIMWCNTs was synthesized by an in-situ polymerization. In this synthesis, initially $f$ MWCNTs $(1 \%)$ were dispersed in water and then ultrasonicated for $30 \mathrm{~min}$. Then organic acid DBSA $(1.5 \mathrm{M})$ was added with vigorous stirring. The precooled solution of aniline monomer $(0.55 \mathrm{M})$ was added to the above dispersion with constant stirring. Finally, the oxidising agent ammonium persulphate $\left(\mathrm{NH}_{4}\right)_{2}$ $\mathrm{S}_{2} \mathrm{O}_{8}(0.55 \mathrm{M})$ was added drop wise over a period of $30 \mathrm{~min}$ with constant stirring to initiate the polymerization of aniline. The monomer to oxidizing agent ratio was kept as 1:1. After $2 \mathrm{hr}$, the dark suspension turned green, indicating the formation of the conducting emeralidine salt phase of the polyaniline. The reaction was continued under stirring for $24 \mathrm{hr}$ at room temperature, in order to ensure the complete polymerization of aniline. The greenish black precipitate obtained was filtered and washed thoroughly with deionized water to remove the impurities of unreacted monomer, excess acid, and oxidant, and then the product was dried in an oven at $60^{\circ} \mathrm{C}$ for $24 \mathrm{~h}$. The free flowing greenish black powder of PANI-MWCNTs nanocomposite was then subjected for physicochemical characterization.

\section{iv) Characterization}

UV-Vis spectra of PANI-MWCNTs were recorded using Perkin-Elmer spectrophotometer in the range of 300-1100 nm. FTIR spectra were studied using PerkinElmer Spectrum 2000 spectrophotometer in the range of $400-4000 \mathrm{~cm}^{-1}$. The samples were prepared in the pellet form using spectroscopic grade $\mathrm{KBr}$ powder. The morphology of the nanocomposite was studied using Fieldemission scanning electron microscopy (FESEM; Hitachi S-4800 II).

\section{Results and Discussion}

Figure 1. shows the UV-Visible absorption spectra of PANI-MWCNTs nanocomposite recorded in the range of $300-1100 \mathrm{~nm}$.

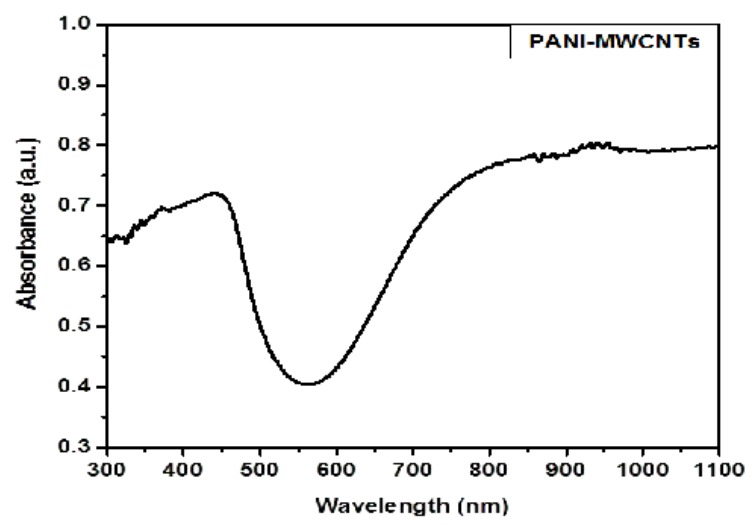

Figure 1. UV-Vis. Spectra of Polyaniline MWCNTs nanocomposite

The as synthesized nanocomposite shows the presence of small peak at $350 \mathrm{~nm}$, a sharp peak appearing at $420 \mathrm{~nm}$ and an increasing 
absorption at $820 \mathrm{~nm}$. The peak at $320 \mathrm{~nm}$ corresponds to the $\pi-\pi^{*}$ transition of the benzenoid rings. While, the shoulder at $420 \mathrm{~nm}$ represents polaronic peak representing the protonation of the polymer, and the sharp peak at $820 \mathrm{~nm}$ showing free carrier tail, confirms the presence of conducting emeraldine salt phase of the polymer [11].

The detailed structural investigations of PANIMWCNTs nanocomposites was carried out by FT-IR spectroscopy and presented as Figure 2 while, the Table 1 gives the characteristic frequencies of the PANI-MWCNTs nanocomposite. The nanocomposite prepared by in-situ polymerization process shows all the characteristic peaks of polyaniline in its conducting emeraldine salt (ES) phase. However, a new band appearing at $1660 \mathrm{~cm}^{-1}$, which is attributed to the carbonyl stretch of the amide. This clearly indicates that the polyaniline would become covalently functionalized to the MWCNTs via the formation of an amide bond.

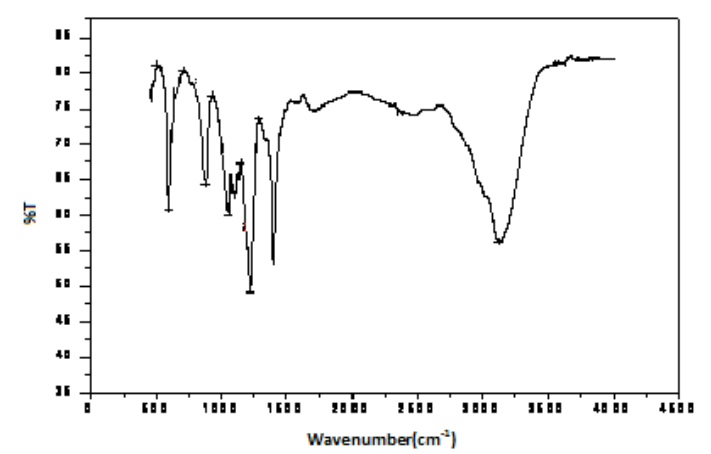

\section{Figure 2 FT-IR spectra of PANI-MWCNT nanocomposite}

Table 1. Characteristic frequencies of Polyaniline Carbon nanotubes nanocomposite

\begin{tabular}{|c|l|}
\hline Wavenumber $\left(\mathrm{cm}^{-1}\right)$ & \multicolumn{1}{|c|}{ Band Characteristics } \\
\hline 878 & $\begin{array}{l}\text { Paradisubstituted } \\
\text { aromatic rings indicating } \\
\text { polymer formation. }\end{array}$ \\
\hline 634 & $\begin{array}{l}\mathrm{C}-\mathrm{H} \text { out of plane } \\
\text { bending vibration. }\end{array}$ \\
\hline 1030 & $\begin{array}{l}-\mathrm{SO}_{3}^{-} \text {group of the } \\
\text { dopant ion }\end{array}$ \\
\hline 1109 & $\begin{array}{l}\text { C-H in plane bending } \\
\text { vibration. }\end{array}$ \\
\hline 1288 & $\begin{array}{l}\text { Aromatic C-N stretching } \\
\text { indicating secondary } \\
\text { aromatic amine group. }\end{array}$ \\
\hline 1460 & $\begin{array}{l}\text { C-N stretching of } \\
\text { benzenoid rings. }\end{array}$ \\
\hline
\end{tabular}

\begin{tabular}{|c|l|}
\hline 1628 & $\begin{array}{l}\text { C-N stretching of quinoid } \\
\text { rings. }\end{array}$ \\
\hline 1660 & $\begin{array}{l}\text { Carbonyl stretch of the } \\
\text { amide }\end{array}$ \\
\hline 3400 & N-H stretching vibration \\
\hline
\end{tabular}

The FE-SEM image of the $f$-MWCNTs and PANI-MWCNTs nanocomposites are shown in Figure 3 ( $a$ and $b$ ). The magnifications were selected such that the morphology could be seen clearly.

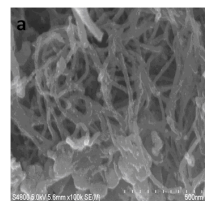

(a)

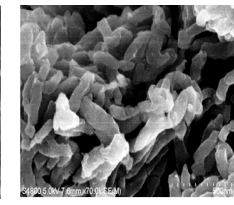

(b)

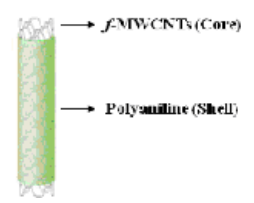

(c)
Figure 3 FE-SEM image of pristine $f-M W C N T s$ (a) PANI-MWCNTs nanocomposite(b) and core shell structure of nanocomposite(c)

Figure 5a, clearly shows the intermingled ropes/tubes with smooth surfaces of $f$-MWCNTs with varying diameter in the range of $\sim 25-35 \mathrm{~nm}$ and length up to few microns. However, in the nanocomposite, a tubular layer of uniform wrapping/coating of PANI is clearly observed on the surface of the $f$-MWCNTs (fig.3b) forming a core - shell structure (fig.3c). As seen in the figure, the diameter of PANI-MWCNTs composites was logically found to be increased as compared to that of the pure/pristine $f$ MWCNTs due to wrapping of the polymer over it.

After successful synthesis and characterization of the PANI-MWCNT nanocomposite the water dispersible and highly transparent nanocomposite was then used for optical $\mathrm{pH}$ sensing.

Figure 4 a shows the dispersion of polyanilineMWCNT nanocomposite at different $\mathrm{pH}$. The nanocomposite solution is dark green in colour at acidic condition $(\mathrm{pH}=1)$ and it is quite obvious because polyaniline at acidic condition are in the conducting emeraldine salt phase which is emeraldine green in colour. As the $\mathrm{pH}$ is increased from acidic to the basic side there is dramatic decrease in the intensity of the green colour of the polyaniline in the nanocomposite. At highly basic condition $(\mathrm{pH}=12)$ the colour of the nanocomposite solution turned to dark/intense blue indicating the transformation of polyaniline emeraldine salt (conducting) phase to its pernigraniline base (insulating) form. 


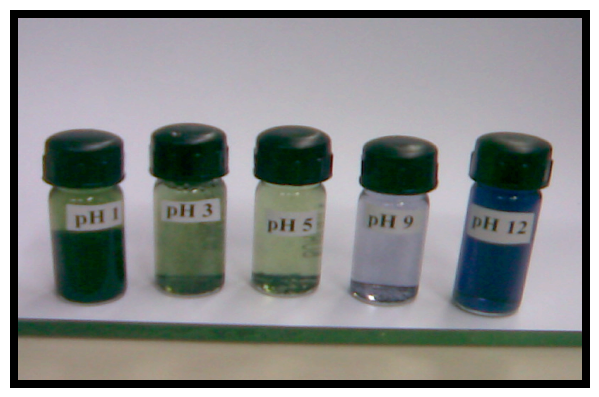

(a)
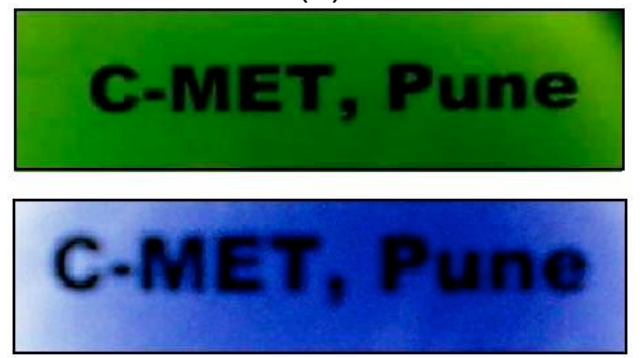

(b)

Figure 4 (a) photograph of Polyaniline MWCNTs nanocomposite dispersion in water at different $\mathrm{pH}, \quad$ (b) Polyanine-MWCNTs nanocomposite thin film coated on glass substrates at two extreme $\mathrm{pH}(\mathrm{pH}=1$ and $\mathrm{pH}=12)$

At intermediate $\mathrm{pH}$ values of 5 and 9 which is nearer to neutral $\mathrm{pH}$, there is a slight tinge of green and blue colour to the nanocomposite solution respectively. This $\mathrm{pH}$ dependent change in the oxidation state of the polyanilineMWCNT nanocomposite which is very easily reflected by the change in the colour (optical change) can be efficiently used for the development of optical $\mathrm{pH}$ sensing measurements. Also, we have coated this nanocomposite dispersion with two extreme $\mathrm{pH}$ on the microscopic glass slides (Fig.4 b) where, the transparency and uniformity of this nanocomposite film is clearly observed. The film is highly transparent with more than $80 \%$ transmittance. It is also possible to make the miniaturized optical sensor device using PANIMWCNT nanocomposite coated optical fibers. The efforts towards the development of this type of device are in progress in our laboratory.

\section{Conclusions}

Highly transparent and water soluble nanocomposite of conducting polymer, Polyaniline (PANI) and Multi-walled Carbon Nanotubes (MWCNTs) was prepared by an insitu chemical polymerization method using sulphonic acid as a dopant.. The synthesized nanocomposite was characterized by using spectroscopic (UV-Vis and FT-IR) analysis. The synthesized nanocomposite was then successfully used for the determination of $\mathrm{pH}$ of the solution and can be considered as a potential candidate for optical $\mathrm{pH}$ sensing.

\section{Acknowledgements}

Authors acknowledge the financial support from Department of Information Technology (DIT) New Delhi. Authors also would like to thank Dr. D. P. Amalnerkar, Executive Director, C-MET for the encouragement throughout this work.

\section{References}

[1] J. Y. Kim, S. I. Han, S. H. Kim, Crystallization behaviors and mechanical properties of poly(ethylene 2,6-naphthalate)/multiwall carbon nanotube nanocomposites, Polym. Eng. Sci. 47, 1715-1723 (2007); doi: 10.1002/pen.20789

[2] J. Y. Kim, H. S. Park, S. H. Kim, Unique nucleation of multi-walled carbon nanotube and poly(ethylene2,6-naphthalate)nanocomposites during non-isothermal crystallization,Polymer, 47 , 1379-1389(2006):doi: 10.1016/j.polymer.2005.12.042,

[3] Y.H.Shin,J.W.Song, E. S. Lee, C. S. Han, Imaging characterization of carbon nanotube tips modified using a focused ion beam, Appl. Surf. Sci. 253,6872-6877(2007); doi: 10.1016/j.apsusc.2007.01.135,

[4] Y. Cao, P. Smith, A. J. Heeger, Counter-ion induced processibility of conducting polyaniline and of conducting polyblends of polyaniline in bulk polymers, Synth. Met., 48, 91-97 (1992); doi: 10.1016/0379-6779(92)90053-L,

[5] A. G. MacDiarmid, J. C. Chiang, A. F. Richter, A.J. Epstein, Polyaniline: a new concept in conducting polymers, Synth. Met., 18, 285-290 (1987); doi; 10.1016/0379-6779(87)90893-9.

[6] T. W. Ebbesen, H. J. Lezec, H. Hiura, J. W. Bennett, H. F. Ghaemi, T. Thio, Electrical conductivity of individual carbon nanotubes, Nature , 382, 54-56 (1996); doi: 10.1038/382054a0

[7] M. J. Esplandiu, V. G. Bittner, K. P. Giapis, C. P. Collier, Nanoelectrode Scanning Probes from Fluorocarbon-Coated Single-Walled Carbon Nanotubes,Nano Letter 4, 1873-1879 (2004); doi: 10.1021/nl048991s

[8] R. H. Baughman, A. A. Zakhidov, W. A. d. Heer, Science, Carbon Nanotubes The Route Toward Applications 297, 787-792 (2002); doi: 10.1126/science.1060928

[9] T. M. Wu, Y. W. Lin, Doped polyaniline/multiwalled carbon nanotube composites: Preparation, characterization and properties, Polymer, 47, 3576-3582 (2006); doi: 10.1016/j.polymer.2006.03.060.

[10] Z. Wei, M. Wan, T. Lin, L. Liming Dai, Polyaniline Nanotubes Doped with Sulfonated Carbon Nanotubes Made Via a Self-Assembly Process, Adv. Mater, 15, 136-139 (2003); doi: 10.1002/adma.200390027.

[11] M. V. Kulkarni, A. K. Viswanath, Scanning Electron Microscopy, Spectroscopy, and Thermal Studies of Polyaniline Doped with Various Sulfonic AcidsJ. Macromol. Sci. Part A. Pure and Applied Chem., 41, 1173-1186 (2004); doi: 10.1081/MA-200026566 